\title{
Aggressive natural killer-cell neoplasm presenting in the marrow: a report of two cases including one with gains of chromosomes $4 q$ and $9 p$
}

\author{
Jie-Yang Jhuang ${ }^{1}$, Alexandra Clipson ${ }^{2}$, Yen-Chuan Hsieh ${ }^{3}$, Chun-Chieh Yang ${ }^{4}$, Sheng-Tsung Chang ${ }^{3,5}$,
} Ming-Qing Du ${ }^{2}$ and Shih-Sung Chuang ${ }^{3,6^{*}}$

\begin{abstract}
Aggressive nature killer (NK)-cell neoplasm includes aggressive NK-cell leukemia (ANKL) and extranodal NK/T-cell lymphoma (ENKTL), nasal type. ANKL is rare and is characterized by a systemic neoplastic proliferation of NK-cells, usually with a leukemic presentation. ENKTL is a predominantly extranodal lymphoma, occurring mainly in the upper aerodigestive tract. Both are aggressive neoplasms strongly associated with Epstein-Barr virus (EBV). Here we report two patients with aggressive NK-cells neoplasms localized in the bone marrow (BM) who presented as prolonged fever, anemia, and thrombocytopenia. Both were treated initially as infectious disease. Imaging studies revealed splenomegaly without any nodular lesion or lymphadenopathy. BM examination revealed extensive involvement by EBV-positive NK-cells in both cases. Staging workup including nasal examination/biopsy was negative. Both patients passed away in a month. One case showed gains of chromosomes $4 q$ and $9 p$ by array comparative genomic hybridization. Both tumors were diagnostically challenging due to the unusual clinical presentation and absence of leukemic change, tumor mass or lymphadenopathy. Our cases demonstrate that lymphoma should be considered in patients with fever of unknown origin and bone marrow aspiration/biopsy should be performed as early diagnosis and novel therapeutic regimens may benefit these patients.
\end{abstract}

Keywords: Aggressive natural killer-cell leukemia, Bone marrow, Epstein-Barr virus, Extranodal natural killer/T-cell lymphoma, Primary bone marrow lymphoma, T-cell lymphoma

\section{Background}

In the 2008 WHO classification, aggressive nature killer (NK)-cell neoplasms include 2 entities: aggressive NKcell leukemia (ANKL) and extranodal NK/T-cell lymphoma, nasal type (ENKTL) [1, 2]. ANKL is rare and characterized by a systemic neoplastic proliferation of NK-cells almost always associated with Epstein-Barr virus (EBV), usually with a leukemic presentation, and an aggressive clinical course. ANKL affects mostly young

\footnotetext{
* Correspondence: cmh5301@mail.chimei.org.tw

${ }^{3}$ Department of Pathology, Chi-Mei Medical Centre, 901 Chung-Hwa RoadYung-Kang District, Tainan, Taiwan

${ }^{6}$ Department of Pathology, College of Medicine, National Taiwan University and Taipei Medical University, Taipei, Taiwan

Full list of author information is available at the end of the article
}

to middle-aged patients and is more common among Asians. ENKTL is a predominantly extranodal lymphoma occurring mainly in upper aerodigestive tract. It is associated with EBV and has a strong ethnic and geographic predilection, being most common among Asians and Native Americans. The distinction between prototypic ANKL and ENKTL is usually straight forward, yet rare cases with overlapping features may exist. Molecular features have been reported to be different between these entities [3]. Here, we report two cases of aggressive NK-cell neoplasms involving BM but without leukemic phase or extranodal involvement. Furthermore, one case showed gains of chromosomes $4 \mathrm{q}$ and $9 \mathrm{p}$ by array comparative genomic hybridization (aCGH). 


\section{Case presentation}

Case 1 was a 55 -year-old Taiwanese female presenting on Oct $18^{\text {th }}, 2009$ with abdominal pain and fluctuating fever for several weeks. There was no prior systemic disease, B symptoms, lymphadenopathy, or skin rashes. Hemograms showed normocytic anemia (hemoglobin at $8.6 \mathrm{~g} / \mathrm{dL}$ ), thrombocytopenia (platelet count at $37,000 / \mu \mathrm{L}$ ), and normal white counts without blasts or leukemic cells. Whole body gallium scan revealed increased uptake only at the bilateral lower lung fields but not in the nasopharynx or elsewhere including spleen. Computed tomography $(\mathrm{CT})$ scans of the chest revealed mixed consolidation and ground-glass opacity over bilateral lower lung zones without mass lesions. Empirical antibiotics were administered but in vain. Bone marrow (BM) biopsy was performed on the $9^{\text {th }}$ admission day and ENKTL with extensive marrow involvement was diagnosed. Her serum lactate dehydrogenase (LDH) level was elevated at $395 \mathrm{IU} / \mathrm{L}$ (reference: 85-227). Abdominal CT scans were negative for lymphadenopathy or mass lesion in the liver or spleen. Nasal endoscopy and biopsy were negative. Her serum EBV viral load was 130,000 copies/ml. A stage IVB disease with ECOG performance status score of 1 and IPI score of 2 was diagnosed. She received one course of chemotherapy with vincristine and prednisolone but developed metabolic acidosis. Unfortunately, her clinical condition deteriorated rapidly and she died of septic shock with multiple organ failure, five weeks after admission and three weeks after diagnosis.

Table 1 summaries the pathological and immunophenotypic features. By flow cytometry, the marrow lymphocytes expressed CD2, CD56 and cytoplasmic CD3 but not surface CD3, CD5, CD7, CD8 or terminal deoxynucleotidyl transferase, indicating an NK-cell phenotype (Fig. 1a-c). The marrow trephine was hypercellular with a diffuse infiltrate of atypical small to medium-sized lymphocytes accompanied by stromal fibrosis (Fig. 1d and e). Immunohistochemically, the atypical lymphocytes expressed CD3 (Fig. 1f), CD45 and T-cell intracellular antigen (TIA)-1 (Fig. 1g) but not CD2, CD4, CD5, CD7, CD8, CD20, CD30, CD56, $\beta F 1$, T-cell receptor (TCR) $-\gamma$ or granzyme B. EBV in situ hybridization (EBER) showed positive signals in around $70 \%$ tumor cells (Fig. 1h). For Infinium genotyping assay, $200 \mathrm{ng}$ DNA isolated from marrow aspirate was hybridized to the HumanCytoSNP-12 Beadchip (Illumina) according to the manufacturer's instructions. Data were analyzed using the genotyping module of the GenomeStudio software (version 1.9.0; Illumina). Copy-number analysis was performed using the cnvPartition copy-number variation (CNV) analysis plug-in for GenomeStudio software (version 3.2.0; Illumina). A confidence value of greater than 100 was used to determine true copy number changes. The copy number analysis indicated a gain of 1 copy at
Table 1 Pathological, immunophenotypical and genotypical findings

\begin{tabular}{lll}
\hline & Case 1 & Case 2 \\
\hline Cell size & Small to medium & Small to medium \\
Tumor necrosis & Absent & Present \\
CD2 & - & - \\
CD3 & + & + \\
SCD3 (FCM) ${ }^{\text {a }}$ & - & ND \\
CD4 & - & - \\
CD5 & - & - \\
CD7 & - & + \\
CD8 & - & + \\
CD16 & ND & - \\
CD30 & ND & - \\
CD56 & - & - \\
CD56 (FCM) & + & ND \\
TIA-1 & + & + \\
Granzyme B & - & - \\
$\beta F 1$ & - & - \\
TCR- $\gamma$ & - & Polyclonal \\
TCR-GR & Polyclonal & \\
\hline
\end{tabular}

${ }^{\mathrm{a}} \mathrm{FCM}$, flow cytometric immunophenotyping using bone marrow aspirate Abbreviations: ND, not done; SCD3, surface CD3; TCR-GR, T-cell receptor gene rearrangement

chromosome 4:83644676 - 4:190742692 (CNV Confidence: 22884.3; Fig. 1i) and a gain of 1 copy in chromosome 9:46587 - 9:21925855 (CNV Confidence: 1630.9) and 9:22098574 - 9:33228189 (CNV Confidence: 2317.0; Fig. 1j) B allele frequency (BAF) plot in both panels demonstrated an altered pattern of BAF $(0.0,0.33,0.67$ and 1.0), while $\log \mathrm{R}$ ratio plot displayed no apparent alteration. This was most likely due to the presence of mosaicism in the region of chromosomal gain [4].

Case 2, a 63-year-old Taiwanese male, presented with abdominal fullness for five days on Sep 25 2013. Physical examination revealed a flat abdomen devoid of tenderness, rebounding pain or lymphadenopathy. Hemograms showed anemia (hemoglobin at $7.4 \mathrm{~g} / \mathrm{dL}$ ), thrombocytopenia (platelet count at $85,000 / \mu \mathrm{L}$ ), and normal white count with presence of myelocytes and nucleated red blood cells (NRBC). There were no blasts or leukemic cells. Laboratory examination revealed elevated LDH level (512 IU/L), abnormal liver and renal function tests, hyperlactatemia, and abnormal inflammatory parameters. CT scans revealed a grade 1 laceration of the slightly enlarged spleen with hemoperitoneum and contrast extravasation without any hepatic lesion or lymphadenopathy. Trans-arterial embolization restored the hemodynamic status with stable hemoglobin level. During hospitalization, lactic acidosis worsened. BM biopsy 


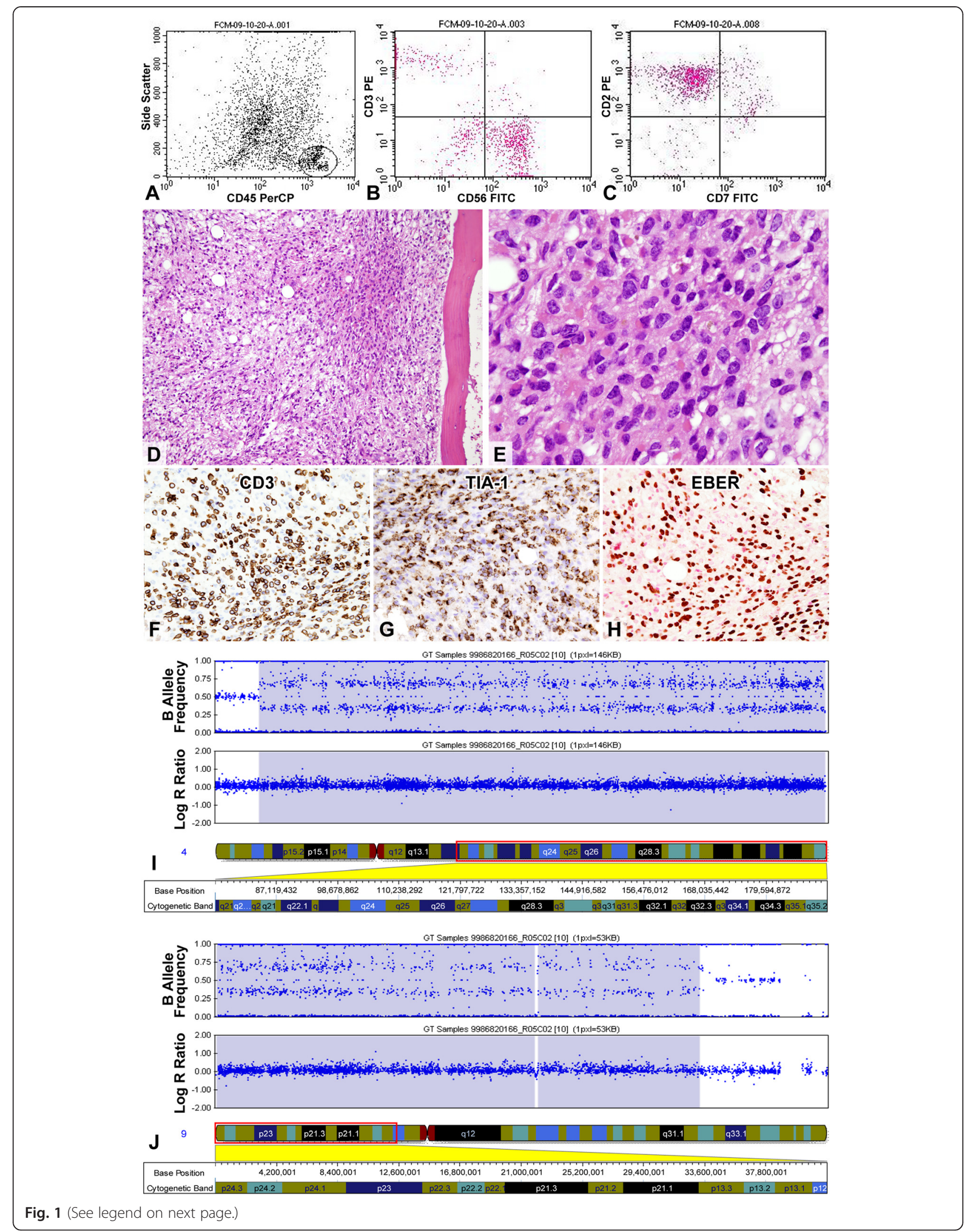


(See figure on previous page.)

Fig. 1 Case 1. Flow cytometric analysis of the marrow aspirate shows that the gated atypical lymphocytes express surface CD2 and CD56 but not CD3 or CD7 (a-c). In addition, they express cytoplasmic CD3 but not TdT, indicating an NK-cell phenotype. Marrow trephine shows a hypercellular marrow with extensive infiltration by small to medium-sized atypical lymphocytes (d and e, HE stains; original magnifications $\times 200$ and $\times 1000$, respectively). The tumor cells express cytoplasmic CD3 (f), TIA-1 $(\mathbf{g})$ and EBER $(\mathbf{h})$. Analyses of genomic copy number alteration using the HumanCytoSNP-12 Beadchip (Illumina). i and j j, These two panels show evidence of gain of one copy at 4q21-q35 (i) and 9p24-p13 (j) respectively. B allele frequency (BAF) plot in both panels demonstrates an altered pattern of BAF $(0.0,0.33,0.67$ and 1.0), which is highlighted in a blue background. Log $R$ ratio plot displays no apparent alteration and this is most likely due to the presence of mosaicism in the region of chromosomal gain

was performed on the $30^{\text {th }}$ admission day and a diagnosis of ENKTL was made. Serology tests showed elevated EBV-CA IgG, EBV-CA IgA, and EBNA IgG. The serum EBV viral load was 60,000copies/ml. Examination of the upper airway by an otorhinolaryngologist with a nasal biopsy was negative. His ECOG performance status score deteriorated to 4 , and he subsequently developed acute oliguric renal failure and hepatic failure. He received only supportive treatment and passed away on the $34^{\text {th }}$ day, one week after pathological diagnosis.

Marrow biopsy of Case 2 showed extensive coagulative necrosis with a remaining small proportion of viable cells at the periphery of the marrow core (Fig. 2a and b). Morphologically, it was difficult to identify the atypical lymphoid cells (Fig. 2c). Immunohistochemical stain with CD3 (Fig. 2d) highlighted the small to medium-sized, polymorphous lymphocytes, including those in the necrotic areas. These cells also expressed CD7, CD8, and TIA-1
(Fig. 2e) and were positive for EBER (Fig. $2 \mathrm{f}$ and g) but not CD2, CD4, CD5, CD56, $\beta$ F1 or TCR- $\gamma$. Clonality study for TCR $-\gamma$ chain gene was polyclonal for both cases using our previously described protocols [5]. There was no fresh/ frozen tissue for copy number analysis.

\section{Discussion}

Primary BM lymphoma (PBML) is rare with poorly defined clinicopathological features. Martinez et al. proposed the following criteria for PBML: isolated BM infiltration with no evidence of nodal or extranodal disease and the exclusion of leukemias/lymphomas that are considered to be primarily involving BM [6]. Both of our patients presented as fever of unknown origin (FUO), anemia, and thrombocytopenia and were treated as infectious disease until BM examination. One main question is, if the bone marrow involvement in our cases represent (i) ANKL, (ii) disseminated ENTKL with

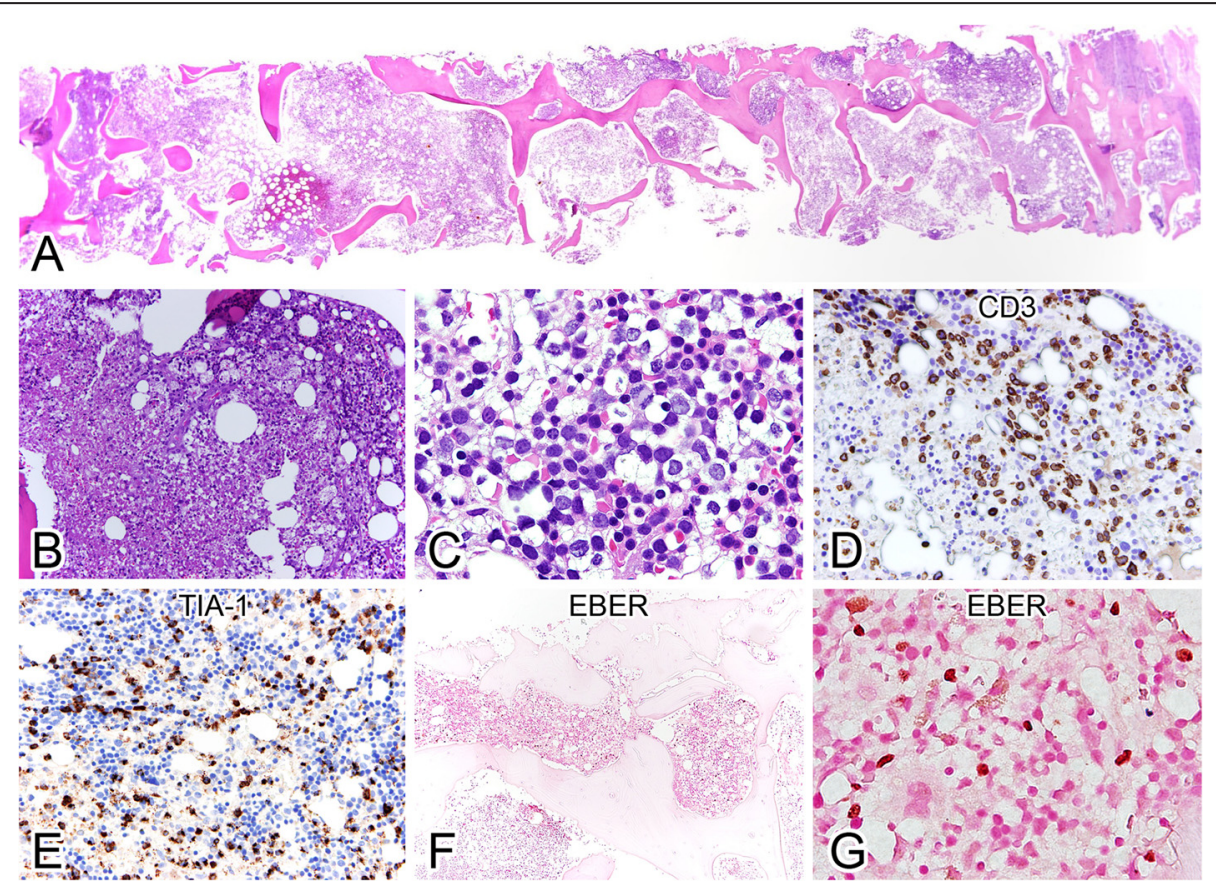

Fig. 2 Case 2. Scanning (a) and medium-powers (b) of the marrow core show extensive coagulative necrosis with scanty viable cells in the right-side end of the core. Even in the high-power view it is difficult to confidently identify the small to medium-sized atypical lymphocytes among the other hematopoietic cells (c). The atypical lymphocytes are highlighted by immunohistostaining with CD3 (d) with irregular nuclear contours and TIA-1 (e). The neoplastic cells are positive for EBV by in situ hybridization (EBER; $\mathbf{f}$ and $\mathbf{g}$, low and high-power views, respectively) 
secondary marrow involvement, or (iii) PMBL. i) ANKL occurs mainly in young adults and shares the same immunophenotype and EBV association with ENKTL. Unlike our cases, patients with ANKL usually have skin rashes, lymphadenopathy, and hepatosplenomegaly and most importantly, leukemic cells in PB [7]. ii) ENKTL with marrow involvement usually occur metachronously or concomitantly with involvement of other anatomical sites such as the aerodigestive tract $[8,9]$. In our patients, examination of the upper aerodigestive tract with biopsy was negative. Neither patient had cutaneous or gastrointestinal lesions. Although CT scans revealed splenomegaly in both cases, there was no nodular lesion/mass or lymphadenopathy. Positron emission tomography (PET)-CT is currently the standard for detecting lymphoma but was not performed in our patients [10]. However, both patients received Gallium scan as part of infectious/inflammatory source workup. Although less sensitive, gallium scans were negative in both patients. iii) Accordingly, our cases fit the criteria of PBML of NK-cell origin. Nasal ENKTL may show minimal involvement of the BM and $\mathrm{PB}$ at disease dissemination, but extensive marrow involvement is extremely rare [11]. The other differential diagnosis is intravascular lymphoma (IVL), frequently of B-cell phenotype with rare examples of T- and/or NK-cell lineage; and the extremely rare cases with EBV association may share similar immunophenotype with ENKTL [12, 13]. Absence of intravascular pattern in our cases argued against the diagnosis of IVL.

There are only a few reports on genome-wide genetic alterations of aggressive NK-cell neoplasms. Nakashima et al. compared ANKL $(n=10)$ with ENKTL $(n=17)$ using array CGH [3]. The recurrent alterations of ANKLs were gain of $1 \mathrm{q}$ and loss of $7 \mathrm{p} 15.1-\mathrm{p} 22.3$ and 17p13.1, while that of ENKTL were gain of $2 \mathrm{q}$, and loss of 6q16.1-q27, 11q22.3-q23.3, 5p14.1-p14.3, 5q34-q35.3, 1p36.23-p36.33, 2p16.1-p16.3, 4q12, and 4q31.3-q32.1. They considered ANKL and ENKTL distinct entities based on different genetic alterations. Recently the same group lumped ANKL $(\mathrm{n}=9)$ and ENKTL $(\mathrm{n}=27)$ together and identified two $6 \mathrm{q} 21$ regions that were most frequently deleted (14 of 39 or $36 \%$ ) using oligo-array CGH and gene-expression profiling [14]. They identified several candidate tumor-suppressor genes that were down-regulated in these regions and their subsequent investigations suggested that two of these suppressor genes, PRDM1 and FOXO3, were considered playing an important role in the pathogenesis of NK-cell neoplasms. However, the genetic alterations in our Case one (Chromosomes 4 and 9) were distinct from that in these 2 reports. Studies on more cases are warranted to elucidate the pathogenesis of this rare group of disease.

\section{Conclusions}

In brief, we describe the clinicopathological findings of two aggressive NK-cell neoplasms as PBML with poor outcome, partly due to a delay in diagnosis. Patients with this aggressive neoplasm may benefit from early and accurate diagnosis and administration of novel therapeutic regimens such as SMILE (L-asparaginase, methotrexate, ifosphamide, etoposide, and dexamethasone) protocol, which could overcome the effects of increased $\mathrm{P}$-glycoprotein by the neoplastic NK-cells through the expression of the multi-drug resistance (MDR) gene.

\section{Consent}

The patients have signed consent forms for reporting their diseases.

\section{Abbreviations}

aCGH: Array comparative genomic hybridization; ANKL: Aggressive NK-cell leukemia; BAF: B allele frequency; BM: Bone marrow; CNV: Copy-number variation; CT: Computed tomography; EBER: EBV in situ hybridization; EBV: Epstein-Barr virus; ENKTL: Extranodal NKTT-cell lymphoma; LDH: Lactate dehydrogenase; NK: Natural killer; PBML: Primary bone marrow lymphoma; PET: Positron emission tomography; TCR: T-cell receptor.

\section{Competing interests}

The authors declare that they have no competing interests.

\section{Authors' contributions}

SSC conceived and oversaw the study. CCY provided the cases, AL and YCH did the experiments. JYJ, MQD, STC, and SSC analyzed the data. JYJ, MQD, and SSC wrote the manuscript. All authors approved the manuscript.

\section{Acknowledgement}

This work was supported by Leukaemia Lymphoma Research, U.K. (to Prof. MQ Du) and Ministry of Science and Technology, Taipei, Taiwan (to Prof. SS Chuang).

\section{Author details}

${ }^{1}$ Department of Anatomic Pathology, Far Eastern Memorial Hospital, New Taipei City, Taiwan. ${ }^{2}$ Department of Pathology, University of Cambridge, Cambridge, UK. ${ }^{3}$ Department of Pathology, Chi-Mei Medical Centre, 901 Chung-Hwa RoadYung-Kang District, Tainan, Taiwan. ${ }^{4}$ Departments of Critical Care Medicine and Pathology, Chi-Mei Medical Center, Tainan, Taiwan. ${ }^{5}$ Department of Nursing, National Tainan Institute of Nursing, Tainan, Taiwan. ${ }^{6}$ Department of Pathology, College of Medicine, National Taiwan University and Taipei Medical University, Taipei, Taiwan.

Received: 22 May 2015 Accepted: 18 June 2015

Published online: 04 July 2015

\section{References}

1. Chan JKC, Jaffe ES, Ralfkiaer E, Ko Y-H. Aggressive NK/T-cell Iymphoma. In: Swerdlow SH, Campo E, Harris NL, Jaffe ES, Pileri SA, Stein H, et al., editors. WHO classification of tumours of haemtopoietic and lymphoid tissues. Lyon: IARC; 2008. p. 276-7.

2. Chan JKC, Quintanilla-Martinez L, Ferry JA, Peh S-C. Extranodal NK/T-cell lymphoma, nasal type. In: Swerdlow SH, Campo E, Harris NL, Jaffe ES, Pileri SA, Stein $\mathrm{H}$, et al., editors. WHO classification of tumours of haemtopoietic and lymphoid tissues. Lyon: IARC; 2008. p. 285-8.

3. Nakashima Y, Tagawa H, Suzuki R, Karnan S, Karube K, Ohshima K, et al. Genome-wide array-based comparative genomic hybridization of natural killer cell lymphoma/leukemia: different genomic alteration patterns of aggressive NK-cell leukemia and extranodal Nk/T-cell lymphoma, nasal type. Genes Chromosomes Cancer. 2005;44(3):247-55. doi:10.1002/ gcc.20245.

4. Conlin LK, Thiel BD, Bonnemann CG, Medne L, Ernst LM, Zackai EH, et al. Mechanisms of mosaicism, chimerism and uniparental disomy 
identified by single nucleotide polymorphism array analysis. Hum Mol Genet. 2010;19(7):1263-75. doi:10.1093/hmg/ddq003.

5. Kuo SY, Liu H, Liao YL, Chang ST, Hsieh YC, Bandoh BA, et al. A parallel comparison of T-cell clonality assessment between an in-house PCR assay and the BIOMED-2 assay leading to an efficient and cost-effective strategy. J Clin Pathol. 2011;64(6):536-42.

6. Martinez A, Ponzoni M, Agostinelli C, Hebeda KM, Matutes E, Peccatori J, et al. Primary bone marrow lymphoma: an uncommon extranodal presentation of aggressive non-hodgkin lymphomas. Am J Surg Pathol. 2012;36(2):296-304. doi:10.1097/PAS.0b013e31823ea106.

7. Win KT, Hsieh YC, Yang CC, Chang ST, Su YZ, Tien HF, et al. Rapidly fatal leukemia comprising pleomorphic large granular lymphocytes: a report of 2 cases. Anal Quant Cytopathol Histpathol. 2013;35(4):232-6.

8. Ko YH, Ree HJ, Kim WS, Choi WH, Moon WS, Kim SW. Clinicopathologic and genotypic study of extranodal nasal-type natural killer/T-cell lymphoma and natural killer precursor lymphoma among Koreans. Cancer. 2000;89(10):2106-16.

9. Ng SB, Lai KW, Murugaya S, Lee KM, Loong SL, Fook-Chong S, et al. Nasal-type extranodal natural killer/T-cell lymphomas: a clinicopathologic and genotypic study of 42 cases in Singapore. Mod Pathol. 2004;17(9):1097-107. doi:10.1038/modpathol.3800157.

10. Fujiwara H, Maeda Y, Nawa Y, Yamakura M, Ennishi D, Miyazaki Y, et al. The utility of positron emission tomography/computed tomography in the staging of extranodal natural killer/T-cell lymphoma. Eur J Haematol. 2011;87(2):123-9. doi:10.1111/j.1600-0609.2011.01645.x.

11. Rezk SA, Huang Q. Extranodal NK/T-cell lymphoma, nasal type extensively involving the bone marrow. Int J Clin Exp Pathol. 2011;4(7):713-7.

12. Au WY, Shek WH, Nicholls J, Tse KM, Todd D, Kwong YL. T-cell intravascular lymphomatosis (angiotropic large cell lymphoma): association with Epstein-Barr viral infection. Histopathology. 1997;31(6):563-7.

13. Wu H, Said JW, Ames ED, Chen C, McWhorter V, Chen P, et al. First reported cases of intravascular large cell lymphoma of the NK cell type: clinical, histologic, immunophenotypic, and molecular features. Am J Clin Pathol. 2005;123(4):603-11. doi:10.1309/X597-G3QM-XAFB-CM5V.

14. Karube K, Nakagawa M, Tsuzuki S, Takeuchi I, Honma K, Nakashima Y, et al. Identification of FOXO3 and PRDM1 as tumor-suppressor gene candidates in NK-cell neoplasms by genomic and functional analyses. Blood. 2011;118(12):3195-204. doi:10.1182/blood-2011-04-346890.

\section{Submit your next manuscript to BioMed Central and take full advantage of:}

- Convenient online submission

- Thorough peer review

- No space constraints or color figure charges

- Immediate publication on acceptance

- Inclusion in PubMed, CAS, Scopus and Google Scholar

- Research which is freely available for redistribution 